\title{
Smart Cities: um estudo prospectivo sobre Internet das Coisas (IoT) aplicada ao setor de mobilidade urbana
}

\section{Smart Cities: a prospective study Internet of Things (IOT) applied to the urban mobility sector}

\author{
Josimary Horta de Araujo ${ }^{1}$ \\ Etelvina Maria Marques Moreira ${ }^{1}$ \\ Carla Fernandes Freitas ${ }^{2}$ \\ Francisco Edivaldo Brito de Castro ${ }^{3}$ \\ Andre Luiz Carneiro de Araujo ${ }^{1}$ \\ Tecia Vieira Carvalho ${ }^{4}$ \\ ${ }^{1}$ Instituto Federal do Ceará, Fortaleza, CE, Brasil \\ ${ }^{2}$ Instituto Nacional da Propriedade Industrial, Fortaleza, CE, Brasil \\ ${ }^{3}$ Fundação de Ciência, Tecnologia e Inovação de Fortaleza, Fortaleza, CE, Brasil \\ ${ }^{4}$ Núcleo de Estudos e Pesquisa do Norte e Nordeste, Fortaleza, CE, Brasil
}

\begin{abstract}
Resumo
Em uma sociedade cada vez mais conectada, despontam tecnologias impactantes em todas as áreas do conhecimento. Nessa conjuntura, a Internet das Coisas (IoT) emerge tendo como princípio interconectar coisas e dispositivos inteligentes a uma rede, o que contribui, efetivamente, para o estreitamento da relação homem X máquina. Nesse contexto, o presente artigo tem o propósito de realizar a prospecção tecnológica de soluções de IoT com potencial de aplicação ao setor de Mobilidade Urbana. Para tal, foi realizado um levantamento das patentes depositadas na base de dados do INPI e do PatentInspiration ${ }^{\circledR}$, como também das produções científicas registradas no Portal de Periódicos da Capes e no Google Acadêmico. A prospecção indica uma movimentação nacional crescente de incentivo à criação de ambientes favoráveis ao desenvolvimento de equipamentos inteligentes, por meio da tecnologia IoT. Os resultados demonstram a necessidade de monitoramento e investimentos no desenvolvimento de tecnologias inteligentes no Brasil, para prover soluções inovadoras para problemas urbanos.
\end{abstract}

Palavras-chave: Prospecção Tecnológica. Internet das Coisas. Mobilidade Urbana.

\begin{abstract}
In an increasingly connected society, impacting technologies emerge in all areas of knowledge. At this juncture, the Internet of Things (IoT) emerges with the principle of interconnecting smart things and devices to a network, which effectively contributes to the tightening of the man-machine relationship. In this context, this article aims to carry out the technological prospection of IoT solutions with potential application to the Urban Mobility sector. For this purpose, a survey of the patents filed in the INPI and PatentInspiration ${ }^{\circledR}$ databases, as well as the scientific productions registered in the Capes Journal Portal and in Google Scholar was made. Prospecting indicates a growing national drive to encourage the creation of enabling environments for intelligent equipment development through IoT technology. The results demonstrate the need for monitoring and investments in the development of smart technologies in Brazil to provide innovative solutions to urban problems.
\end{abstract}

Keywords: Technological Prospection. Internet of Things. Urban Mobility.

Área Tecnológica: Cidades Inteligentes. Tecnologia. 


\section{Introdução}

Em 1950, apenas 30\% da população residia em áreas urbanas, atualmente esse número corresponde a $55 \%$ do total da população mundial, um percentual cuja projeção para 2050 deve atingir uma proporção de $68 \%$, o que significa dizer que as taxas de urbanização associadas ao crescimento mundial da população poderão trazer mais de 2,5 bilhões de pessoas para as cidades (ONU, 2018).

A grande quantidade de pessoas convivendo no mesmo espaço traz consigo inúmeros desafios ao ordenamento urbano, entre os quais destacam-se as necessidades relacionadas à infraestrutura, à mobilidade, à ocupação do solo, à energia, à educação, etc. Essa relação não é nenhuma novidade, entretanto, estimativas apontam que até 2030 a população mundial deverá atingir o número de 8,5 bilhões de pessoas, um aumento de 1,4 bilhões, sendo que $96 \%$ do crescimento ocorrerão nas cidades do mundo em desenvolvimento (ONU, 2018). Já no Brasil, o Instituto Brasileiro de Geografia Estatística (IBGE, 2018) revela que, de 208.494.900 habitantes em 2018, o país passará a ter 224.868 .462 habitantes em $2030,7,3 \%$ de crescimento em 12 anos.

Esse ponto de vista implica que os problemas das cidades necessitam de tratamento com urgência, pois tendem a crescer com o aumento populacional que continua se intensificando. $\mathrm{O}$ agravante relacionado a esse aumento, no que diz respeito à mobilidade, é o fato de que historicamente os investimentos de infraestrutura de transportes são tratados despreocupadamente em relação às consequências com a qualidade da vida urbana, inclusive quanto à própria eficiência da mobilidade (PINHEIRO; FRISCHTAK, 2016).

No Brasil, têm especial destaque as questões relacionadas à mobilidade urbana, não apenas em decorrência dos problemas inerentes a essa temática, como também aos relacionados à imperiosa necessidade de os municípios do país, com mais de 20 mil habitantes, elaborarem um plano de mobilidade urbana compatível com o plano diretor municipal, conforme preconizado pela Lei Federal n. 12.587, aprovada em 2012.

O estudo intitulado "Future of Urban Mobility" corrobora a importância da temática mobilidade, apontando uma previsão elevada de investimento nesse setor. Segundo esse estudo, o setor de Mobilidade Urbana é a principal área das cidades que atrai o maior volume de investidores. Constata-se também que o setor de Mobilidade Urbana é aquele que predominantemente possui a maior necessidade de investimentos nas cidades (AUDENHOVE et al., 2014).

Desse modo, torna-se imprescindível discutir o incremento do nível de inteligência desses centros urbanos de modo que sejam capazes de constituir cidades mais eficientes, sustentáveis e colaborativas, cujo funcionamento dos diferentes campos se dê de forma inovadora e integrada. De acordo com Ferreira (2016, p. 72), "A solução de problemas inerentes ao ambiente urbano torna a adoção de ferramentas tecnológicas mais sofisticadas um caminho natural para a gestão das cidades".

Uma cidade inteligente, ou, conforme se encontra disponível na literatura, uma Smart City é definida como ambiente urbano que faz uso de diversas Tecnologias de Informação $e$ Comunicação para otimizar a administração, o acesso e a utilização dos serviços disponíveis, buscando de maneira constante e interativa soluções para as necessidades de seus habitantes (FERREIRA, 2016). 
É fundamental para qualquer exercício de avaliação perante o universo de cidades inteligentes conceituar inteligência. Uma análise recente de conceito de "cidade inteligente" demonstrou pontos de vistas diferenciados quando se trata do termo cidade inteligente. O Quadro 1 traz algumas definições de cidades inteligentes.

Quadro 1 - Conceitos de cidade inteligente

\begin{tabular}{|c|c|}
\hline Autor & CONCEITO \\
\hline AHVENNIEMI et al. (2017) & $\begin{array}{l}\text { As cidades vêm se esforçando no sentido de tornarem-se mais } \\
\text { inteligentes. Porém, é importante considerar que os objetivos de cidades } \\
\text { inteligentes e cidades sustentáveis estão estreitamente interligados. }\end{array}$ \\
\hline Cunha et al. (2016) & $\begin{array}{c}\text { Uma cidade inteligente é aquela que supera os desafios do passado e conquista } \\
\text { o futuro, utilizando a tecnologia como meio para prestar de forma mais } \\
\text { eficiente os serviços urbanos e melhorar a qualidade de vida dos cidadãos. }\end{array}$ \\
\hline $\begin{array}{l}\text { Marsal-Llacuna, Colomer- } \\
\text { Llinàs e Meléndez- } \\
\text { Frigola (2015) }\end{array}$ & $\begin{array}{l}\text { Melhorar o desempenho urbano usando dados, informações e TICs, } \\
\text { para fornecer serviços mais eficientes aos cidadãos, monitorar e } \\
\text { otimizar a infraestrutura existente, incrementar a colaboração entre } \\
\text { diferentes atores econômicos e encorajar modelos de negócios } \\
\text { inovadores tanto no setor privado quanto no setor público. }\end{array}$ \\
\hline $\begin{array}{l}\text { Papa, Gargiulo e } \\
\text { Galderisi (2013) }\end{array}$ & $\begin{array}{l}\text { A essência das futuras cidades inteligentes é baseada na ideia da } \\
\text { coordenação e integração de tecnologias que foram desenvolvidas } \\
\text { separadamente, mas que possuem claras sinergias em sua operação } \\
\text { e precisam ser casadas com uma abordagem bottom-up. }\end{array}$ \\
\hline Lazaroiu e Roscia (2012) & $\begin{array}{l}\text { Uma nova forma de viver e considerar a cidade, apoiada em TICs } \\
\text { que podem ser integradas em uma solução para gestão da energia, } \\
\text { água, segurança pública, mobilidade e gestão de resíduos. }\end{array}$ \\
\hline Eger (2009) & $\begin{array}{l}\text { Toma decisões de forma consciente para implementar tecnologias de forma } \\
\text { agressiva para resolver seus problemas sociais e necessidades de negócios, } \\
\text { fazendo uso dessas tecnologias também como oportunidade para reconstruir e } \\
\text { renovar o sentimento de lugar e de orgulho cívico, promover o desenvolvimento } \\
\text { e o crescimento econômico, emprego e melhoria da qualidade de vida. }\end{array}$ \\
\hline
\end{tabular}

Fonte: Elaborado pelos autores deste artigo

Como pode-se observar são muitos os conceitos de cidades inteligentes que variam desde um conceito que foca principalmente no tecnológico até conceitos que colocam cidades inteligentes com foco na questão humana.

O conceito de Tecnologia da Informação e Comunicação envolve um amplo espectro de designações e aplicações. Neste trabalho, esse conceito referenciará os meios tecnológicos utilizados por pessoas e organizações para acessar informações por meio da telecomunicação (ZHANG; AIKMAN; SUN, 2008 apud FERREIRA, 2016). Para tanto, utilizou-se a Internet of Things (IoT) ou - em tradução livre para o Brasil, "Internet das Coisas", considerada o estágio avançado da internet no qual os objetos, as máquinas e os equipamentos se relacionam com outros objetos e com seres humanos por intermédio de dispositivos computacionais capazes de conexão e de comunicação. De acordo com Alecrin (2016), Internet das Coisas pode ser descrito como um cenário em que numerosos objetos do seu dia a dia estarão conectados à internet e se comunicando mutuamente.

Segundo Evans (2011), entre 2008 e 2009, pela primeira vez na história, o número de dispositivos conectados por habitante se aproximou de dois dispositivos (igual exatamente a 1,84). Ao assumir a lógica de pensar evansiana, pode-se visualizar um cenário de previsão para 
o aumento de dispositivos conectados na internet e sua relação com o número de habitantes em escala mundial, fatores capazes de incentivar inovações disruptivas com a IoT. Percebe-se também, pelos estudos de Evans (2011), que, após 2010, o número de dispositivos conectados e sua relação com a população mundial dobra a cada cinco anos, com previsão de chegar em 2020 a 50 bilhões de dispositivos conectados e cada habitante utilizando aproximadamente sete dispositivos.

Ademais, a criação do Plano Nacional da Internet das Coisas, conhecido como Plano de IoT, pelo governo brasileiro, em junho de 2019, corrobora a utilização dessa tecnologia como indicador neste estudo. O Decreto n. 9.854/2019 veio com a finalidade de implementar e de desenvolver a Internet das Coisas no país, além de promover o desenvolvimento sustentável e competitivo da economia brasileira. O Ministério da Ciência, Tecnologia, Inovações e Comunicações (MCTIC, 2019) declara que

O Plano Nacional de IoT é uma iniciativa do Ministério da Ciência, Tecnologia, Inovações e Comunicações (MCTIC), Ministério da Economia e do Banco Nacional de Desenvolvimento Econômico e Social (BNDES) em conjunto com a sociedade civil - empresas, academia, agências de fomento e outros órgãos - para garantir que o Brasil se beneficie da tecnologia de IoT.

Estudo realizado pelo Instituto Brasileiro de Administração Municipal (IBAM), denominado "Mobilidade e política urbana: subsídios para uma gestão integrada", define mobilidade como "[...] um conjunto estruturado de modos, redes e infraestruturas que garante o deslocamento das pessoas na cidade e que mantém fortes interações com as demais políticas urbanas" (MACÁRIO apud IBAM, 2005, p. 10).

Ao abordar estratégias de mobilidade a serem aplicadas a cidades inteligentes, Ferreira (2016) elenca sete alternativas tecnológicas:

1) pedágio inteligente (controle de tráfego de automóveis em partes específicas da cidade);

2) monitoramento geoespacial das redes de transportes ou georreferenciamento de dados (coleta, armazenagem e tratamento das informações);

3) redes de transporte público integradas (integração entre os mais diversos modos de transporte e a unificação do sistema de pagamento);

4) estacionamentos inteligentes (integração do uso de sensores, painéis de sinais de trânsito e aplicativos na otimização da procura por vagas);

5) semáforos e sinais de trânsito automatizados (softwares de inteligência artificial e sensores para otimização do fluxo de veículos e pessoas, por meio do processamento digital da informação);

6) compartilhamento de automóveis e bicicletas (aluguel de veículos por curtos espaços de tempo);

7) veículos elétricos integrados e autônomos (automóveis sem o comando humano executando a navegação com o auxílio de ferramentas como GPS e sensores).

De acordo com Hancke e Silva (2012), existem exemplos em que sensores poderiam ser utilizados em várias áreas de cidades inteligentes, como em Smart Transportation, em que 
sensores são usados para determinar a intensidade e a velocidade do trânsito contribuindo para o planejamento de novas vias e aperfeiçoamento de semáforos, que também podem ser inteligentes. Em Smart Eletricity and Water Distribution (distribuição inteligente de eletricidade e água) é possível utilizar sensores para identificar vazamentos de água ou cortes na eletricidade. Também é possível melhorar o consumo desses recursos utilizando sensores para coletar dados por meio de medidores inteligentes.

Para Hancke e Silva (2012) e Neirotti et al. (2014), existem vários exemplos de como o IoT pode auxiliar no desenvolvimento de soluções para diferentes problemas, entre eles: gerenciamento de lixo, congestionamentos, consumo de energia, monitoramento de poluição sonora, etc. Para esses autores, IoT foi definido como um novo paradigma que colocará dispositivos do dia a dia na internet por meio de componentes de rede e protocolos próprios. Muitos desses problemas fazem parte tanto das áreas apresentadas por Hancke e Silva (2012) quanto dos domínios apresentados por Neirotti et al. (2014). Assim, embora haja certa diferença entre as definições, é possível perceber que há um acordo entre pesquisadores de que cidades inteligentes e IoT têm papel importante em áreas como trânsito, segurança, consumo de energia e de água, etc.

Diante do exposto, pretende-se com este estudo, por meio da aplicação da ferramenta de prospecção tecnológica, visualizar o panorama de soluções de rede de Internet das Coisas (IoT) com potencial de aplicação em mobilidade urbana, assim como identificar os principais atores envolvidos no desenvolvimento dessas tecnologias, na condução de alternativas capazes de incrementar os investimentos nesse segmento, como também o de gerar impacto social referente à melhoria dos níveis de qualidade de vida da população, além de contribuir na construção de políticas públicas municipais.

\section{Metodologia}

Foi realizado um estudo prospectivo de soluções tecnológicas de IoT com potencial de aplicação no setor de Mobilidade Urbana. No primeiro momento, foi feito um levantamento dos processos de pedidos de patentes depositados em âmbito nacional e internacional no período de 2009 a 2019. Justifica-se esse recorte temporal pelo estudo de Evans (2011), quando o número de dispositivos conectados por habitante se aproximou a dois dispositivos.

Nas buscas de pedidos internacionais de depósitos de patentes utilizou-se a plataforma PatentInspiration ${ }^{\circledR}$, sistema de busca e análise de patentes com informações de mais de 76 milhões de patentes depositadas em diversos países. Para as buscas de pedidos nacionais, foi utilizada a base de patentes do Instituto Nacional da Propriedade Industrial (INPI), sistema de busca e análise com informações de patentes no Brasil.

A busca da produção acadêmica foi efetuada nas plataformas Google Acadêmico e no Portal de Periódicos da Coordenação de Aperfeiçoamento de Pessoal de Nível Superior (CAPES), biblioteca virtual que reúne mais de 45 mil títulos, com 12 bases dedicadas exclusivamente a patentes.

Em ambos os processos de busca foram utilizadas palavras-chave em português e em inglês relacionadas aos segmentos de Mobilidade Urbana conforme mostra a Figura 1.

A fim de obter melhores resultados, foram aplicados nas buscas os operadores booleanos, de trucagem e de proximidade AND, OR, ( ), Asterisco (*) e aspas ("”). Todas as buscas foram 
efetuadas na modalidade avançada, em cada uma das bases consultadas e realizadas entre 5 de junho a 20 de julho de 2019.

A utilização inicial de uma grande variedade de palavras-chaves nas buscas nas bases de produção científica e de patentes teve como objetivo encontrar o maior número de resultados possível relacionados à tecnologia de IoT e ao setor de Mobilidade Urbana. Na busca inicial foram escolhidos os termos de buscas "Urban Mobility OR Urban Traffic" e "Iot OR Internet of Things AND Urban Mobility OR Urban Traffic".

O emprego dos termos "Urban Mobility OR Urban Traffic" na busca inicial foi intencional, com o intuito de obter um panorama geral das produções científicas e de patentes relacionadas ao segmento de Mobilidade Urbana, de 2009 até o momento. A definição da expressão de busca "Iot OR Internet of Things AND Urban Mobility OR Urban Traffic" teve por finalidade delimitar o levantamento de produções científicas relacionadas ao segmento de Mobilidade Urbana associado à Internet das Coisas.

Para aprimorar as buscas tecnológicas, foi efetuada, em seguida, a análise pela Classificação Internacional de Patentes (CIP), de modo a viabilizar a organização dos documentos das patentes por área tecnológica e facilitar o acesso às informações tecnológicas e legais neles contidos (MARTINEZ; SANTOS; WINTER, 2015).

Para incrementar a pesquisa e apontar uma linha de interesse pela temática, procedeu-se a busca na ferramenta Google Trends que apresenta o histórico de pesquisa de um termo particular, quando procurado em toda a Web, em determinado espaço de tempo e em vários idiomas.

Figura 1 - Metodologia para buscas em bases de patentes e acadêmicas
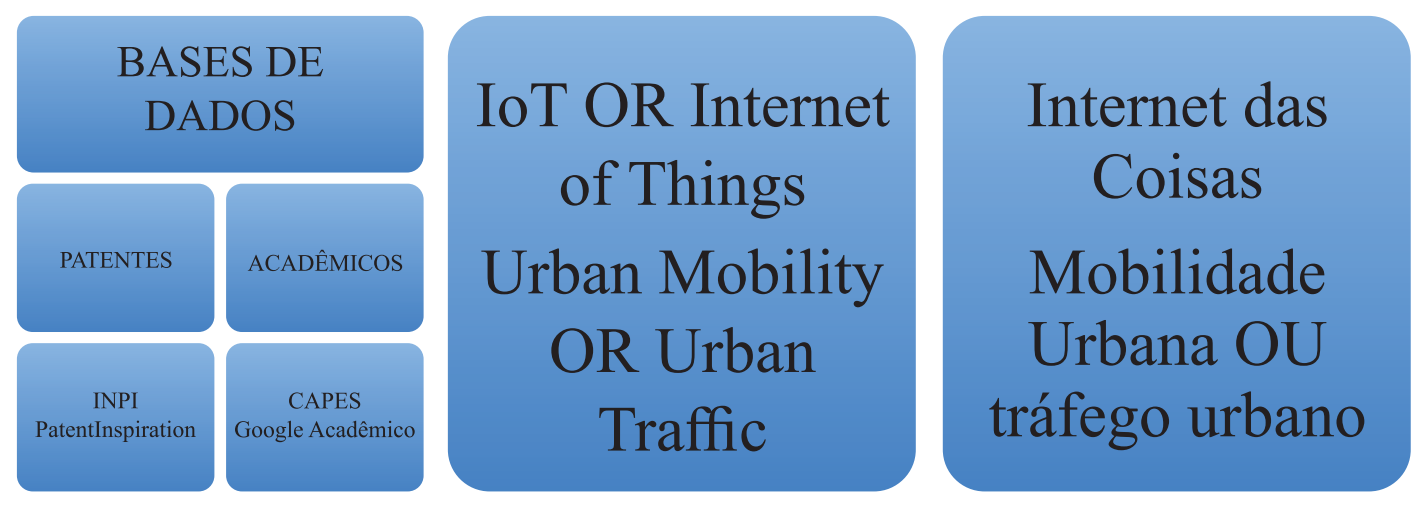

Fonte: Elaborada pelos autores deste artigo

\section{Resultados e Discussão}

Com relação aos resultados obtidos nas buscas de produção científica, verificou-se que a base do Portal de Periódicos da Capes apresentou o maior número de publicações em todas as categorias de pesquisa comparado aos resultados encontrados na base Google Acadêmico. Conforme mostra a Figura 2, a base do Portal de Periódicos da Capes apontou 238.914 resultados envolvendo termos específicos relacionados ao segmento de Mobilidade Urbana que foram: "Urban Mobility OR Urban Traffic" e "Mobilidade Urbana OU Tráfego Urbano", enquanto na base do Google Acadêmico foram encontrados 6.484 resultados. 
Utilizando os termos de buscas "IoT OR Internet of Things" e "Internet das coisas" nas mesmas bases, essa superioridade se confirma com 282.769 resultados encontrados na base do Portal da Capes, enquanto na base do Google Acadêmico só foram obtidos 19.500 resultados.

Com relação à busca de patentes no Brasil e no exterior, ao utilizar os termos "IoT OR Internet of Things", verificou-se a supremacia no número de patentes depositadas na base Patentlnspiration ${ }^{\circledR}$, com 186.222 resultados, enquanto na base nacional do INPI, ao utilizar os termos "Internet das coisas", foram recuperados apenas 72 registros. Ao utilizar os termos "Mobilidade Urbana OU Tráfego Urbano" na base do INPI, obteve-se 25 resultados, enquanto na base PatentInspiration ${ }^{\circledR}$, os resultados para os termos "Urban Mobility OR Urban Traffic" chegaram a 35.472 patentes.

Analisando os resultados encontrados na base nacional de patentes depositadas, é possível afirmar que nos últimos 10 anos o Brasil não tem investido significativamente na proteção de tecnologias ligadas ao sistema IoT. Tampouco no setor de Mobilidade Urbana que mantém dependência tecnológica de outros países, que importam tecnologia, o que eleva o custo dos investimentos e amplia o tempo necessário para implementação de inovações no setor.

Figura 2 - Palavras-chave utilizadas na busca e resultados encontrados - 2009 a 2019

\begin{tabular}{|c|c|c|c|c|}
\hline & \multicolumn{2}{|c|}{ PATENTES } & \multicolumn{2}{c|}{ PERIÓDICOS } \\
\hline Palavras-chave & PatentInspiration & INPI & CAPES & Google Acadêmico \\
\hline IoT OR Internet of Things & 186.222 & 72 & 282.769 & 19.500 \\
\hline Urban Mobility OR Urban Traffic & 35.472 & 25 & 238.914 & 6.484 \\
\hline
\end{tabular}

Fonte: Elaborada pelos autores deste artigo

Inversamente proporcional aos resultados apresentados na Figura 2, verificou-se um número expressivo de pesquisas científicas obtidas na busca nas bases do Portal da Capes e do Google Acadêmico, ao utilizar os termos "IoT OR Internet of Things", "Internet das Coisas", "Urban Mobility OR Urban Traffic" e "Mobilidade Urbana OU Tráfego Urbano", o que demonstra um resultado expressivo na produção de conhecimento no Brasil e no mundo.

Figura 3 - Investimento em Mobilidade Urbana

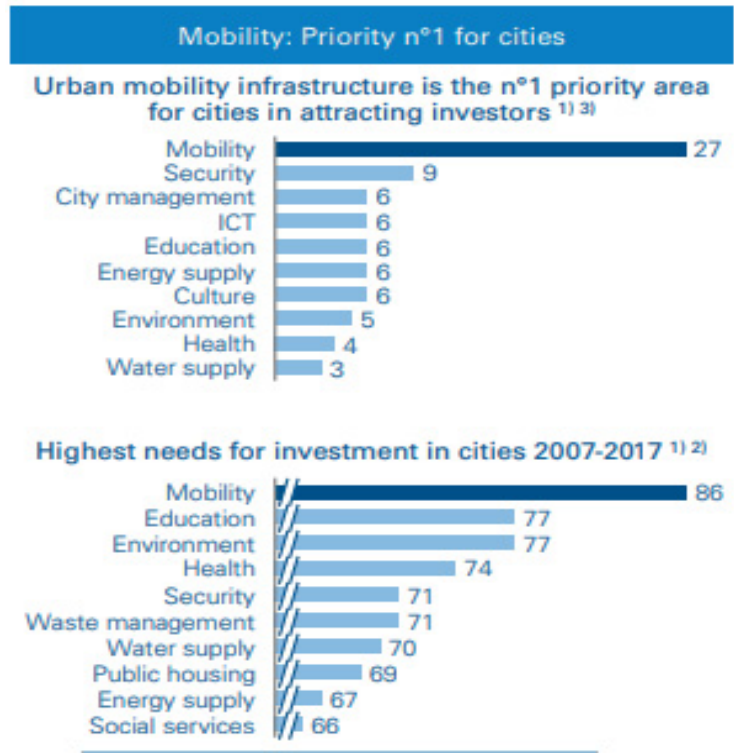

Constant growth of investments in urban mobility Global urban mobility investment volume in bn EUR

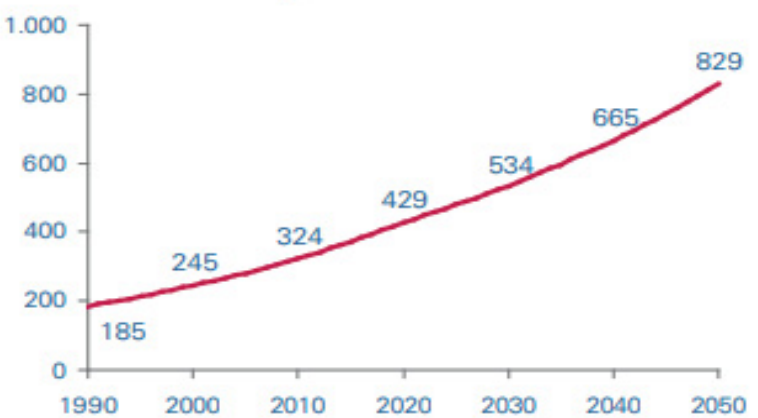

- In 1990 investment amount in global urban mobility equaled 185 bn EUR

- For 2050 the need of $\mathbf{8 2 9}$ bn EUR is being forecasted (growth by the factor 4.5 )

Fonte: Audenhove et al. (2014) 
Conforme apresentado na Figura 3, elaborada com dados extraídos do estudo "Future of Urban Mobility", constatou-se que o setor de Mobilidade Urbana é o principal segmento das cidades que atrai o maior volume de investidores (27\%), enquanto o segundo mais procurado para investimento é o de Segurança. Além disso, constatou-se que o setor de Mobilidade Urbana é aquele que predominantemente possui a maior necessidade de investimentos nas cidades.

Ressalta-se que o estudo detectou um crescimento significativo no volume de investimentos necessários para o setor de Mobilidade Urbana. Em 2010, o investimento global na área urbana ascendeu a 324 bilhões de euros. Em 2050, há previsão de que serão necessários 829 bilhões de euros por ano.

Gráfico 1 - Patentes X Artigos

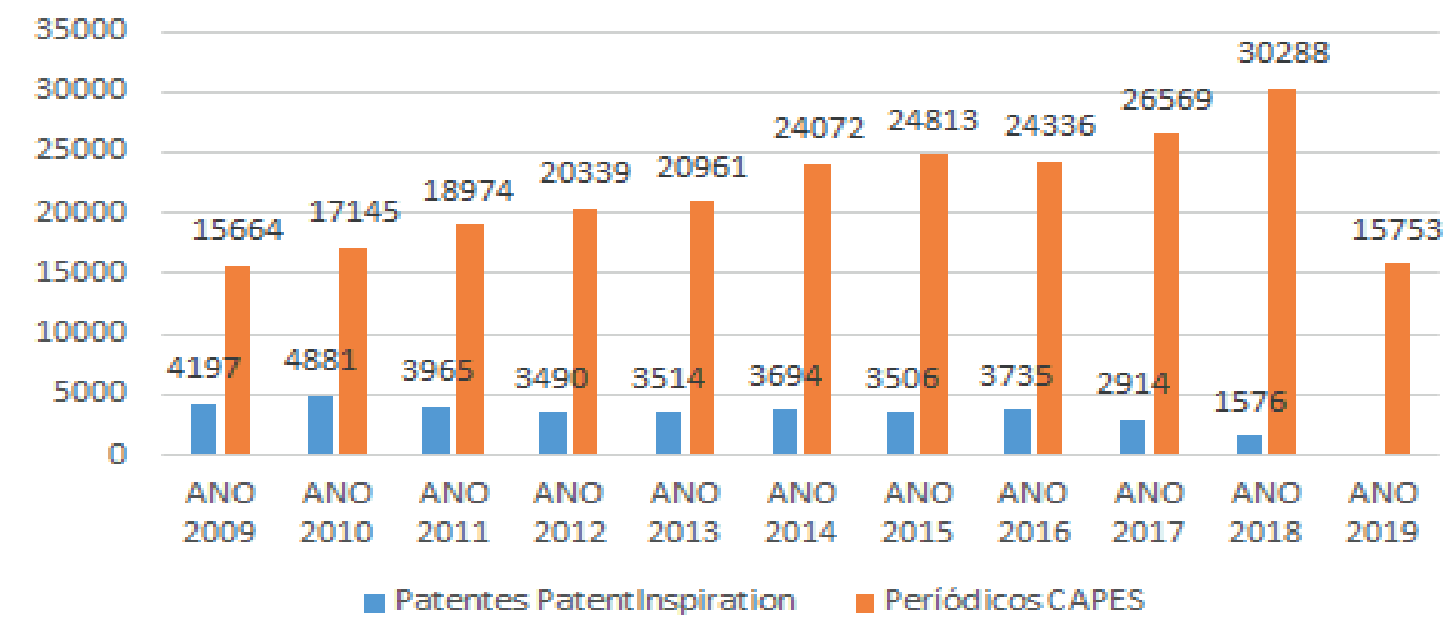

Fonte: Elaborado pelos autores deste artigo

O Gráfico 1 apresenta dados comparativos entre o número de produções científicas e de patentes depositadas de 2009 a 2019, por ano. Verificou-se que o número de artigos de periódicos se sobrepõe ao número de depósitos de patentes, em todos os anos verificados.

Gráfico 2 - Patentes X Artigos - 2009 a 2019

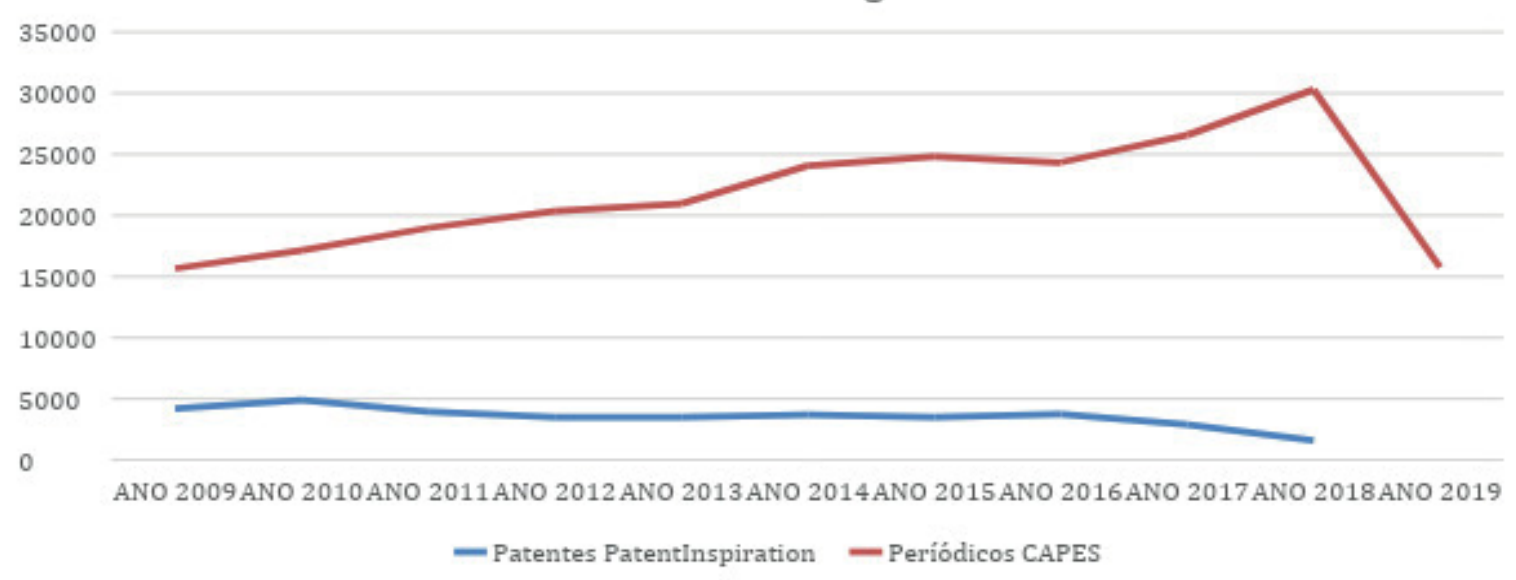

Fonte: Elaborado pelos autores deste artigo 
O Gráfico 2 exibe dados comparativos entre o número de produções científicas e de patentes depositadas de 2009 a 2019. Verifica-se, ao analisar os Gráficos 1 e 2, que a quantidade de artigos excede o número de patentes para os termos "IoT OR Internet of Things", "Internet das coisas", "Urban Mobility OR Urban Traffic" e "Mobilidade Urbana OU Tráfego Urbano", o que demonstra um elevado interesse científico em contraste com a existência de um decréscimo tecnológico no setor de Mobilidade Urbana.

Um dado importante que pode explicar o elevado número de pesquisas acadêmicas se deve à incessante busca de soluções tecnológicas para o setor de mobilidade urbana, tendo em vista o crescimento excepcional da população e, consequentemente, os impactos ambientais desse crescimento. Quanto aos dados de números de patentes depositados, observa-se que, devido ao período de sigilo de 18 meses, os dados nos anos de 2018 e 2019 não estão completos. Considerando a evolução da tecnologia, os resultados possivelmente continuarão estáveis quando esses números forem computados.

$\mathrm{Na}$ análise dos principais depositantes de patentes na base "PatentInspiration ${ }^{\circledR}$ ", mostrados nos Gráficos 3 e 4, observou-se que as empresas com o maior número de patentes depositadas relativas ao setor de Mobilidade Urbana são a Qualcomm, a Ericsson, a Telefon, a Google e a At \& T. Quando empregados os termos de buscas "IoT OR Internet of Things", AND "Urban Mobility OR Urban Traffic", essas mesmas empresas aparecem como as líderes depositantes.

Gráfico 3 - Principais depositantes na base de patente - termos de busca: Urban Mobility OR Urban Traffic

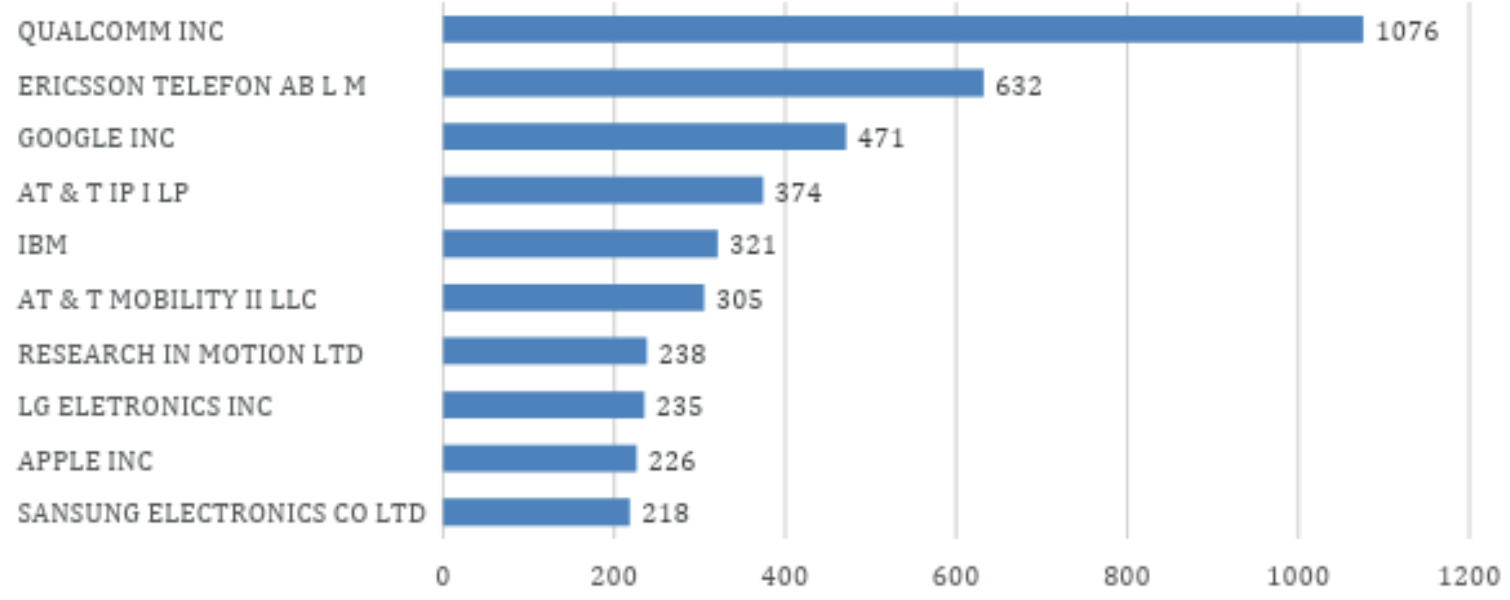

Fonte: Elaborado pelos autores deste artigo

A Qualcomm é uma empresa de componentes e dispositivos, fundada no ano de 1985 por sete inventores graduados no Massachusetts Institute of Technology (MIT). Localizada em San Diego, Califórnia (EUA), possui 224 filiais em todo o mundo. Em 2017, a empresa realizou pesquisa acerca da importância da melhoria das grandes cidades e dos planos de mobilidade urbana. Essa empresa é líder em número de patentes depositadas para ambos os termos de busca, possuindo 1.076 e 544 patentes depositadas, respectivamente.

A Ericsson Telefon, empresa sueca de redes e telecomunicações, fundada em 1876, investe continuamente em pesquisa e seu principal objetivo é a micromobilidade. Ela aparece como a segunda colocada em número de patentes depositadas para ambos os termos de busca, com 632 e 351 patentes nessa ordem. 
Gráfico 4 - Principais depositantes na base de patente - termos de busca: IoT OR Internet of Things AND Urban Mobility OR Urban Traffic

\section{QUALCOMM INC \\ ERICSSON TELEFON AB L M \\ AT \& T IP I LP \\ GOOGLE INC \\ AT \& T MOBILITY II LLC \\ IBM \\ SOROCA ADAM \\ LG ELETRONICS INC \\ GM GLOBAL TECH OPERATIONS INC \\ RAMER JOREY}

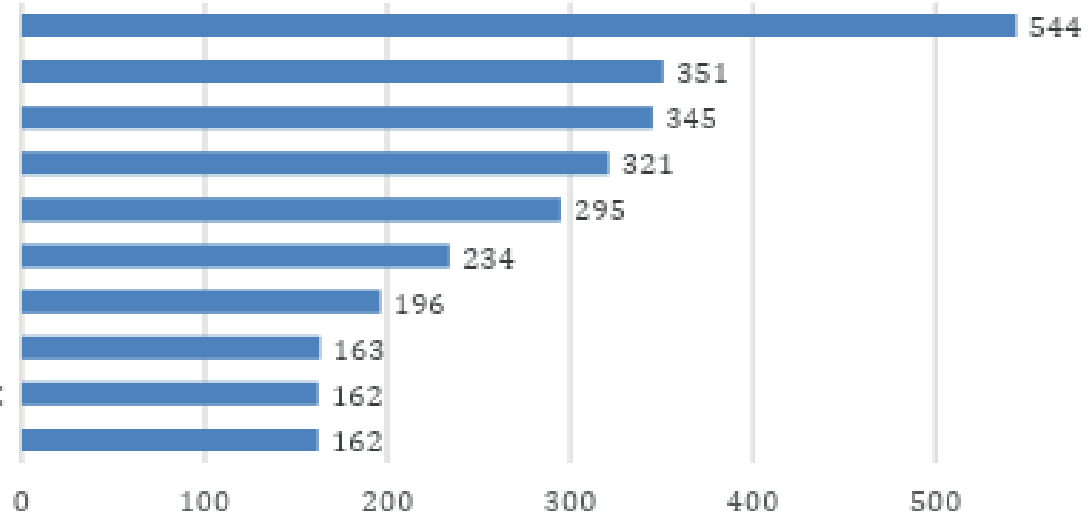

544

600

Fonte: Elaborado pelos autores deste artigo

De acordo com a análise dos resultados das buscas, usando os termos "Urban Mobility OR Urban Traffic", constatou-se que os códigos IPC de maior importância são: H04W4/02 (Serviços que utilizem informação de localização adaptado para rede de comunicação sem fio); G06F17/30 (Equipamentos ou métodos de computação digital ou processamento para recuperação de informações); G08G1/01 (Sistemas de controle de tráfego para veículos rodoviários); e H04L29/08 (Controle de comunicação e de nível de link de dados), conforme apontado no Gráfico 5.

Gráfico 5 - Patentes distribuídas pelo IPC - termo de busca na base "PatentInspiration": Urban Mobility OR Urban Traffic

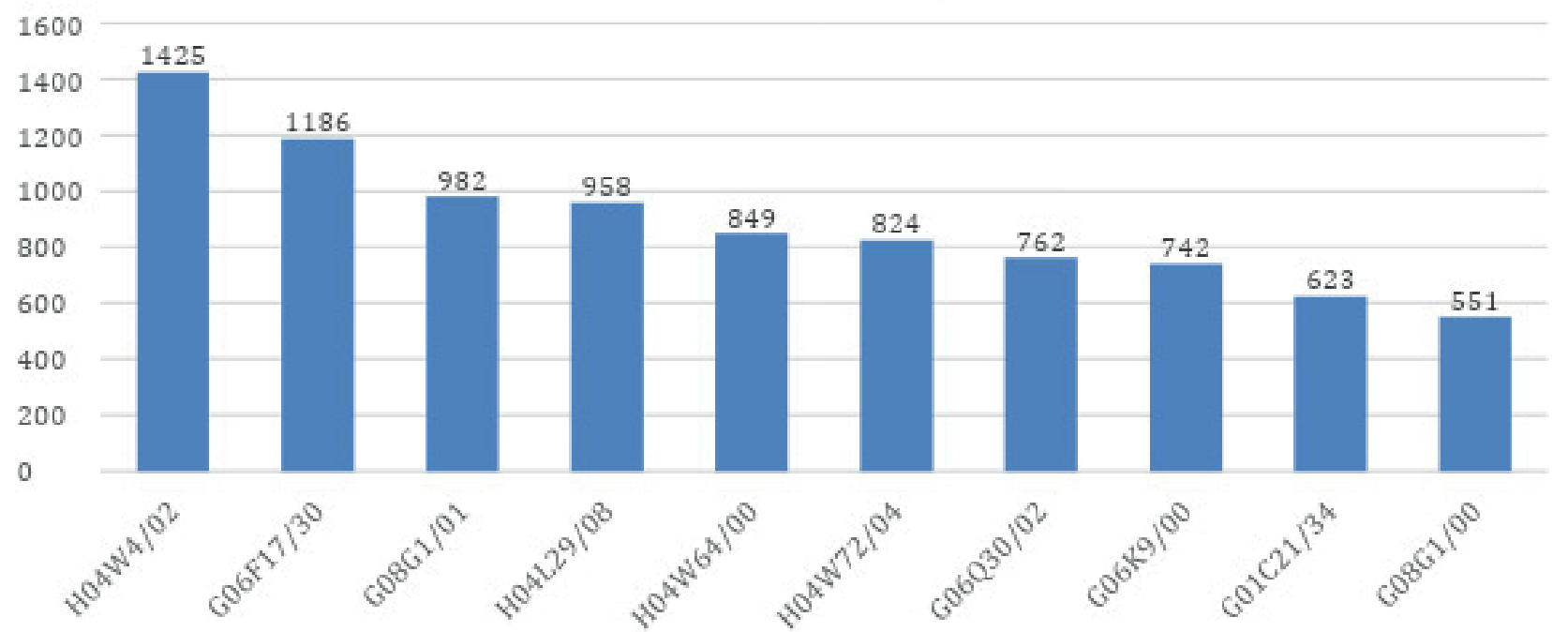

Fonte: Elaborado pelos autores deste artigo

$\mathrm{Na}$ busca, utilizando os termos "IoT OR "Internet of Things", AND "Urban Mobility OR "Urban Traffic", os resultados apontam que os códigos IPC de maior relevância são: G08G1/01 (Sistemas de controle de tráfego para veículos rodoviários); H04W4/02 (Serviços que utilizem informação de localização adaptado para rede de comunicação sem fio); G06F17/30 (Equipamentos ou métodos de computação digital ou processamento para recuperação de informações); 
e H04L29/08 (Controle de comunicação e de nível de link de dados), conforme apresentado no Gráfico 5.

Gráfico 6 - Patentes distribuídas pelo IPC - termo de busca na base "PatentInspiration": IoT OR Internet of Things AND Urban Mobility OR Urban Traffic

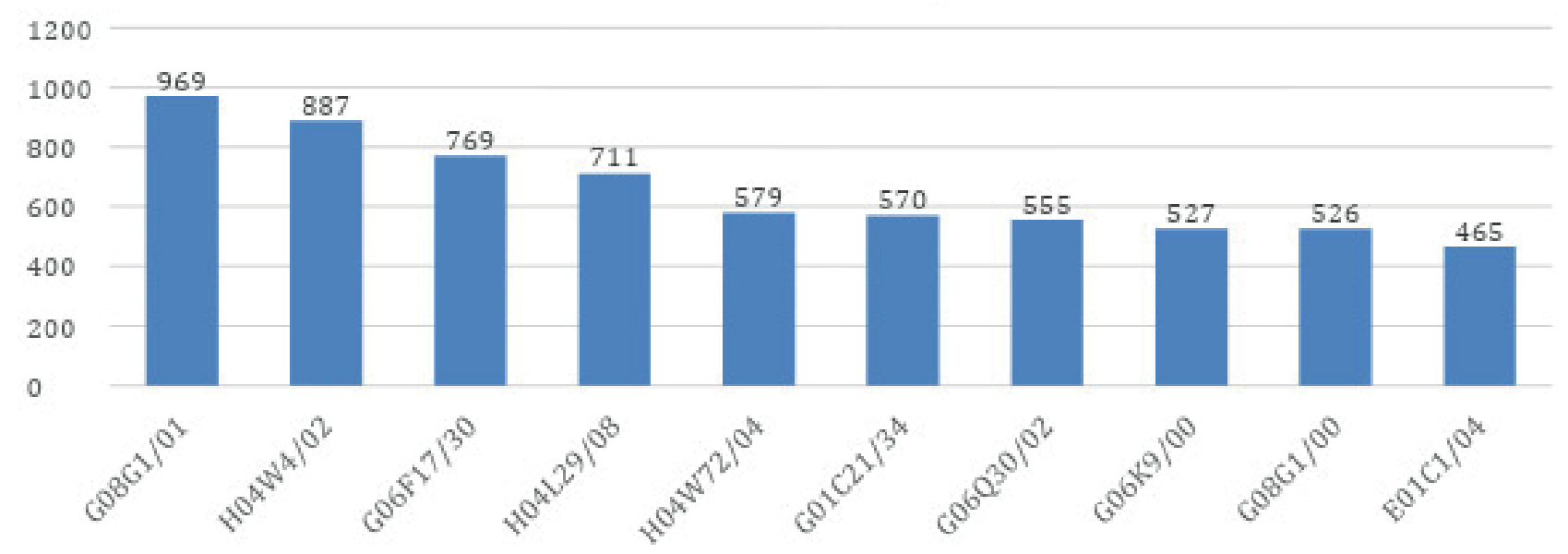

Fonte: Elaborada pelos autores deste artigo

Nas buscas, utilizando os termos "IoT OR Internet of Things" e "IoT OR Internet of Things AND Urban Mobility OR Urban Traffic", observou-se que as quatro principais classificações IPC são as mesmas, com alteração, apenas, na ordem de apresentação. Vale ressaltar que entre as 10 classificações que apresentam o maior número de patentes nas buscas, usando os termos "Urban Mobility OR Urban Traffic", assim como nas buscas com os termos "IoT OR Internet of Things AND Urban Mobility OR Urban Traffic", seis delas pertencem à categoria "G", sendo que as três de maior destaque se referem à subcategoria "G06" (Física utilizando informática, cálculo e contagem).

Gráfico 7 - Visão geral das principais tecnologias - Termo de busca na base de patente "PatentInspiration": IoT OR Internet of Things AND Urban Mobility OR Urban Traffic

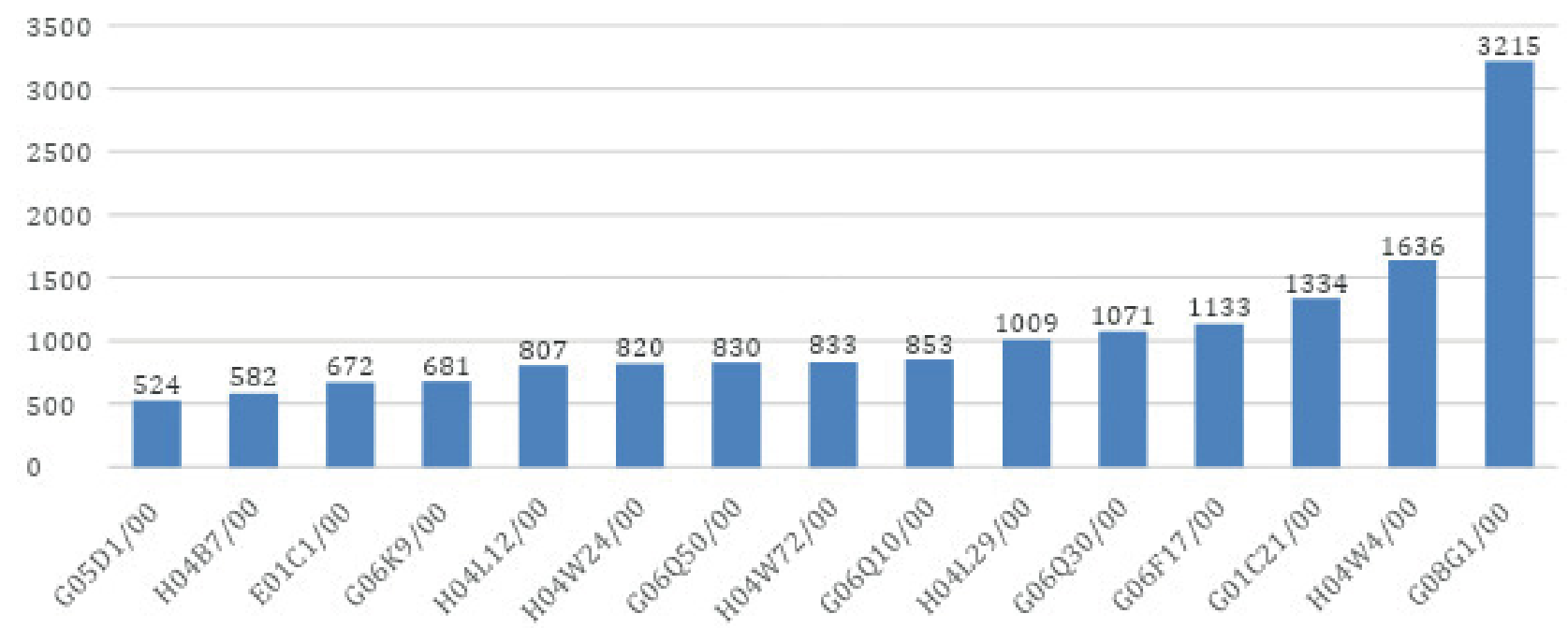

Fonte: Elaborado pelos autores deste artigo 
O Gráfico 7 revela que a produção das patentes está direcionada para duas vertentes: dispositivos conectados e comunicação interveicular. Essas duas linhas de aplicação permitem a identificação de sete cenários tecnológicos para o setor de Mobilidade Urbana: 1) Estacionamento Inteligente; 2) Redes de Transporte Público Integradas; 3) Compartilhamento de Automóveis, bicicletas e patinetes; 4) Semáforos e Sinais de Trânsito Automatizados; 5) Pedágio inteligente; 6) Monitoramento geoespacial de transporte; e 7) Veículos Elétricos, Integrados e Autônomos.

Por meio das informações extraídas da base "PatentInspiration ${ }^{\circledR}$ " e visualizadas na Figura 4, constatou-se a supremacia dos Estados Unidos em relação aos depósitos de patentes desse setor. O país possui 10.174 patentes depositadas nos últimos 10 anos, porém, considerando ao período de sigilo, esses números provavelmente são bem maiores. Esse dado é de suma relevância, pois, além de os Estados Unidos possuírem a maior economia nacional do mundo e uma avançada infraestrutura de transporte, a maior parte dos seus habitantes utiliza o automóvel como principal meio de transporte. Nota-se, ainda, que outros países, como a China, o Japão, o Canadá e a Alemanha, também desenvolvem pesquisas relacionadas ao setor de Mobilidade Urbana e Internet das Coisas, porém em proporção substancialmente inferior.

Figura 4 - Mercados e localização competitiva de acordo com a base de patente "PatentInspiration" (IoT OR Internet of Things AND Urban Mobility OR Urban Traffic)
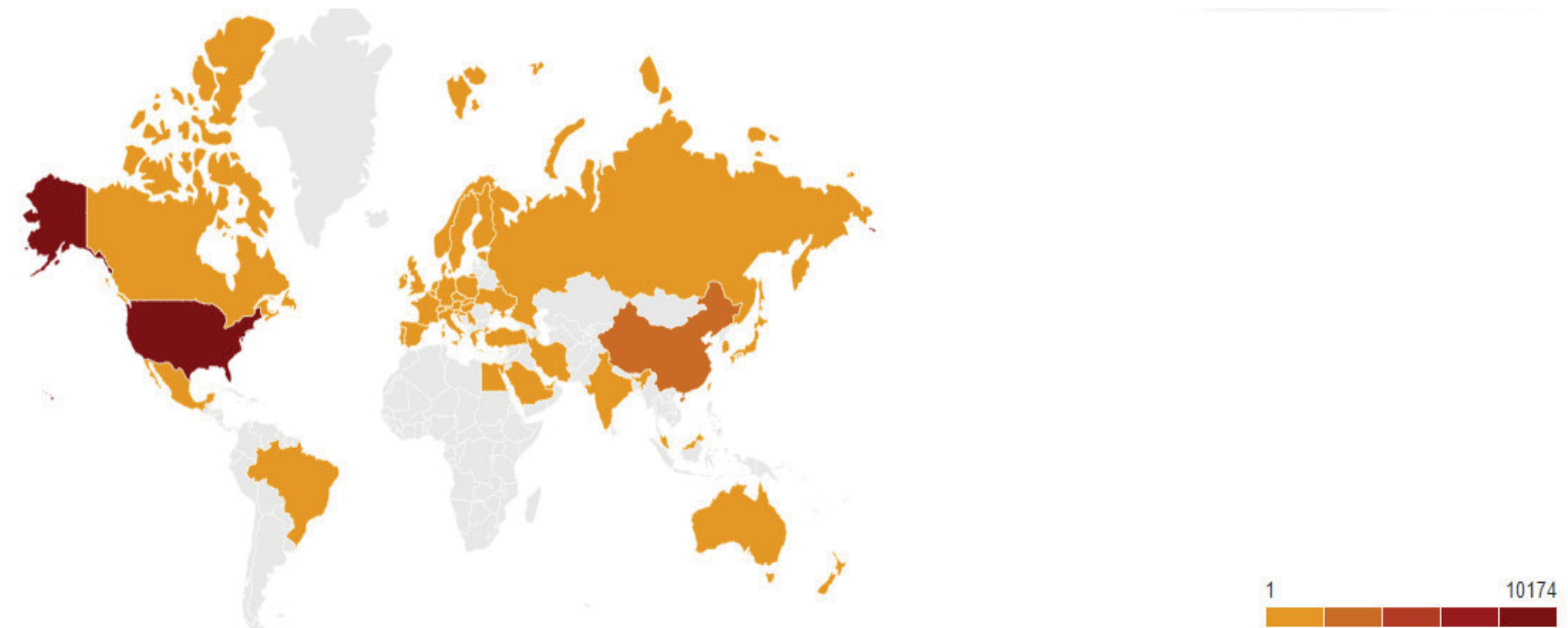

Fonte: Gerado pela ferramenta PatentInspiration ${ }^{\circledR}$

\section{Considerações Finais}

A IoT tem se revelado uma tecnologia de grande valia para a captação, a gestão e a utilização racional das informações por proporcionar uma mudança sem precedentes na interação dos humanos com as máquinas, reduzindo alguns obstáculos produzidos por interfaces não adaptativas a fatores humanos. A redução do tempo de deslocamento dos veículos e o aumento da atratividade pelo transporte coletivo são os principais fatores para a aplicação da IoT no cenário de mobilidade urbana. Esta pesquisa permitiu verificar, no âmbito mundial e nacional, os estudos e as tecnologias desenvolvidas que apresentam relação direta com a Internet das Coisas (IoT) e com os dispositivos e equipamentos utilizados no setor de Mobilidade Urbana. 
As prospecções realizadas demonstram que os Estados Unidos possuem o maior número de depósitos de patentes desse setor, enquanto no Brasil os equipamentos inteligentes, com potencial de modificar de forma significativa o setor de Mobilidade Urbana no país, são incipientes.

Os atuais problemas da mobilidade urbana que poderiam ser resolvidos pela aplicação do conceito de IoT ainda carecem de ferramentas de baixo custo. A realidade da IoT no setor de mobilidade urbana pode ser exemplificada pela presença de sensores para estacionamentos inteligentes e para semáforos e sinais de trânsito automatizados, de sistemas de redes de transporte público integrados e de pedágios inteligentes, além de dispositivos para compartilhamento de automóveis, bicicletas e patinetes para monitoramento geoespacial de transporte e para veículos elétricos, integrados e autônomos.

Os dados apresentados neste estudo, assim como a ausência de pesquisa sobre a temática deste artigo, revelam a necessidade de monitoramento e de investimentos no desenvolvimento de tecnologias inteligentes no Brasil. Também revelam a necessidade de aprofundamento da pesquisa em cada cenário tecnológico apresentado, de modo que a gestão pública possa promover um desenvolvimento ordenado e sustentável dos seus centros urbanos.

Apesar dos baixos índices de inovação existentes no Brasil, observa-se uma movimentação nacional crescente de incentivo à criação de ambientes favoráveis ao desenvolvimento de equipamentos inteligentes, por meio da tecnologia IoT. Com a criação, em junho de 2019, do Plano Nacional de Internet das Coisas, por meio do Decreto n. 9.854/2019, espera-se o estímulo do desenvolvimento nacional por meio da promoção de tecnologias inteligentes que possam atender, entre outros, ao setor de Mobilidade Urbana.

\section{Referências}

AHVENNIEMI, H. et al. What are the differences between sustainable and smart cities? Cities, [S.I.], v. 60, p. 234-245, 2017.

ALECRIN, E. O que é Internet das Coisas (Internet of Things)? Publicado em: 07.03.2016.

Atualizado em: 17.01.2017. Disponível em: https://www.infowester.com/iot.php. Acesso em: 5 jul. 2019.

AUDENHOVE, F. V. et al. The future of urban mobility 2.0: imperatives to shape extended mobility ecosystems of tomorrow. Boston: Arthur D. Little, 2014. Disponível em: https:/www.adlittle. com/sites/default/files/viewpoints/2014_ADL_UITP_Future_of_Urban_Mobility_2_0_Full_study.pdf. Acesso em: 9 maio 2019.

BERGMAN, L.; RABI, N. I. A. (coord.). Mobilidade e política urbana: subsídios para uma gestão integrada. Brasília, DF: Ministério das Cidades; Instituto Brasileiro de Administração Municipal, 2005. Disponível em: http://www.ibam.org.br/media/arquivos/estudos/mobilidade.pdf. Acesso em: 24 jul. 2019.

BRASIL. Decreto n. 9.854, de 25 de junho de 2019. Institui o Plano Nacional de Internet das Coisas e dispõe sobre a Câmara de Gestão e Acompanhamento do Desenvolvimento de Sistemas de Comunicação Máquina a Máquina e Internet das Coisas. Brasília, DF: Presidência da República, [2019]. Disponível em: http://www.planalto.gov.br/ccivil_03/_Ato2019-2022/2019/Decreto/D9854. htm. Acesso em: 15 jun. 2019. 
BRASIL. Lei Federal n.12.587, de 3 de janeiro de 2012. Institui as diretrizes da Política Nacional de Mobilidade Urbana. Brasília, DF: Presidência da República, [2012]. Disponível em: http://www. planalto.gov.br/ccivil_03/_Ato2011-2014/2012/Lei/L12587.htm. Acesso em: 24 jul. 2019.

BRASIL. Lei Federal n. 13.683, de 19 de junho de 2018. Altera a Lei n. 13.089, de 12 de janeiro de 2015 (Estatuto da Metrópole), e a Lei n. 12.587, de 3 de janeiro de 2012, que institui as diretrizes da Política Nacional de Mobilidade Urbana. Brasília, DF: Presidência da República. [2018]. Disponível em: http://www.planalto.gov.br/ccivil_03/_ato2015-2018/2018/Lei/L13683.htm. Acesso em: 24 jul. 2019.

CAPES. Portal de Periódicos. Base de dados on line. [2019]. Disponível em: http://www.periodicos. capes.gov.br/. Acesso em: 15 jun. 2019.

CHIEREGATTI, C. M. A mobilidade urbana de Brasília: um estudo descritivo em comparação com as propostas de uma cidade inteligente. 2016. 65 f. Monografia (Bacharelado em Administração) - Departamento de Administração, Universidade de Brasília, Brasília, 2016. Disponível em: http://bdm.unb.br/bitstream/10483/14004/1/2016_CarolinaMoreiraChieregatti_tcc. pdf. Acesso em: 24 jul. 2019.

CUNHA, M. A. et al. Smart Cities: Transformação Digital de Cidades. 1. ed. São Paulo: Programa Gestão Pública e Cidadania, 2016. v. 1. 161 p.

EGER, J. M. Smart growth, smart cities, and the crisis at the pump a worldwide phenomenon.

I-Ways - The Journal of E-Government Policy and Regulation, [S.l.], v. 32, n. 1, p. 47-53, 2009.

EVANS, D. A. Internet das Coisas: como a próxima evolução da Internet está mudando tudo. San Jose, Canada: Cisco Internet Business Solutions Group (IBSG), 2011. Disponível em: http://www. cisco.com/web/BR/assets/executives/pdf/internet_of_things_iot_ibsg_0411final.pdf. Acesso em: 24 set. 2019.

FERREIRA, A. A. Estratégias e iniciativas para mobilidade em cidades inteligentes. 2016. 78 p. Monografia (Graduação em Engenharia) - Escola de Engenharia, Universidade Federal do Rio Grande do Sul, Porto Alegre, 2016. Disponível em: https://lume.ufrgs.br/handle/10183/159577. Acesso em: 25 abr. 2019.

GOOGLE ACADÊMICO. Base de dados on line. [2019]. Disponível em: https://scholar-googlecom-br.ez138.periodicos.capes.gov.br/scholar?hl=pt-BR\&q=. Acesso em: 15 jun. 2019.

GOOGLE TRENDS. Base de dados on line. [2019]. Disponível em: https:/trends.google.com.br/ trends/?geo=BR. Acesso em: 19 jul. 2019.

HANCKE, G. P.; SILVA, B. C. The role of advanced sensing in smart cities. Sensors (Basel, Switzerland), [S.I.], v. 13, p. 393-425, 2012.

IBAM - MINISTÉRIO DAS CIDADES (Brasil). Mobilidade e política urbana: subsídios para uma gestão integrada. Rio de Janeiro, 2005. Convênio n. 7/2004.

IBGE - INSTITUTO BRASILEIRO DE GEOGRAFIA E ESTATÍSTICA. [2018]. Disponível em: https:// www.ibge.gov.br/apps/populacao/projecao. Acesso em: 14 out. 2019.

INPI - INSTITUTO NACIONAL DA PROPRIEDADE INDUSTRIAL. Base de dados de patentes on line. [2019]. Disponível em: https://gru.inpi.gov.br/pePI/jsp/patentes/ PatenteSearchBasico.jsp. Acesso em: 9 maio 2019. 
LATIPAT. Base de dados on line. [2019]. Disponível em: https://worldwide.espacenet.com/. Acesso em: 10 jul. 2019.

LAZARIOU, G. C.; ROSCIA, M. C. Definition methodology for the smart cities model. Energy, [S.l], v. 47, n. 1, p. 326-332, 2012.

MARSALL-LLACUNA, M. L.; COLOMER-LLINÀS, J.; MELÉNDEZ-FRIGOLA, J. Lessons in urban monitoring taken from sustainable and livable cities to better address the Smart Cities initiative.

Technological Forecasting and Social Change, [S.l.], v. 90, p. 611-622, 2015.

MARTINEZ, M. E. M.; SANTOS, D. A.; WINTER, E. Mapeamento por meio de documentos patentários depositados no Brasil das tecnologias do setor de celulose e papel. O Papel, São Paulo, v. 76, n. 10, p. 77-81, 2015.

MCTIC - MINISTÉRIO DA CIÊNCIA, TECNOLOGIA, INOVAÇÕES E COMUNICAÇÕES. Decreto que institui o Plano Nacional de Internet das Coisas é publicado. Brasília, 26 jun. 2019. Disponível em: http://www.mctic.gov.br/mctic/opencms/salalmprensa/noticias/ arquivos/2019/06/Decreto_que_institui_o_Plano_Nacional_de_Internet_das_Coisas_e_publicado. html?searchRef =Plano\%20Nacional\% $20 \overline{\mathrm{d}}$ \% 20 Internet\%20das\%20Coisas\&tipoBusca $=$ expressaoEx ata. Acesso em: 5 jul. 2019.

NEIROTTI, P. et al. Current trends in smart city initiatives: Some stylised facts. Cities, [S.l.], v. 38, p. 25-36, 2014.

ONU - ASSEMBLEIA GERAL DAS NAÇÕES UNIDAS. Population. [2018]. Disponível em: http:// www.onu-brasil.org.br. Acesso em: 14 out. 2019.

PAPA, R.; GARGIULO, C.; GALDERISI, A. Towards and urban planners perspective os smart city. Tema Journal of Land Use, Mobility and Evironment, [S.l.], v. 6. n. 1, p. 5-17, 2013.

PATENTINSPIRATION. Base de dados on line. [2019]. Disponível em: https://app. patentinspiration.com/\#report/1b7DBd32eC8c/filter. Acesso em: 13 jul. 2019.

PEREIRA, A.; SIMONETTO, E. O. Indústria 4.0: conceitos e perspectivas para o Brasil. Revista da Universidade Vale do Rio Verde, Betim, v. 16, n. 1, jul. 2018. Disponível em: http://periodicos. unincor.br/index.php/revistaunincor/article/view/4938/pdf_808. Acesso em: 5 jul. 2019.

PINHEIRO. A. C.; FRISCHTAK. C. Mobilidade Urbana: desafios e perspectivas para as cidades Brasileiras. 1. ed. São Paulo: Elsevier, 2016.

UNITED NATIONS. Department of Economic and Social Affairs. Population Division. 2018 revision of world urbanization prospects. New York, 2018. Disponível em: https://www.un.org/ development/desa/publications/2018-revision-of-world-urbanization-prospects.html. Acesso em: 24 jul. 2019.

ZHANG, P.; AIKMAN, S. N.; SUN, H. Two Types of Attitudes in ICT Acceptance and Use.

International Journal of Human-Computer Interaction, [S.l.], v. 24, p. 628-648, 2008. 10.1080/10447310802335482. 


\section{Sobre os Autores}

\section{Josimary Horta de Araujo}

E-mail: josimaryhorta@ifce.edu.br

Especialista.

Endereço profissional: Rua Jorge Dumar, n. 1.703, Jardim América, Fortaleza, CE. CEP: 60410-426.

\section{Etelvina Maria Marques Moreira}

E-mail: etelvina@ifce.edu.br

Especialista.

Endereço profissional: Rua Jorge Dumar, n. 1.703, Jardim América, Fortaleza, CE. CEP: 60410-426.

\section{Carla Fernandes Freitas}

E-mail: carlaff@gmail.com

Especialista.

Endereço profissional: Rua Doutor Mário Martins Coelho, n. 36, Aldeota, Fortaleza, CE. CEP: 60170-280.

\section{Francisco Edivaldo Brito de Castro}

E-mail: edivaldobc@gmail.com

Especialista.

Endereço profissional: Rua Leonardo Mota, n. 2.700, Dionísio Torres, Fortaleza, CE. CEP: 60170-176.

\section{Andre Luiz Carneiro de Araujo}

E-mail: andreluiz.ifce@gmail.com

Doutor.

Endereço profissional: Avenida 13 de Maio, n. 2.081, Benfica, Fortaleza, CE. CEP: 60040-215.

\section{Tecia Vieira Carvalho}

E-mail: tecia.carvalho@nepen.org.br

Pós-doutora.

Endereço profissional: Rua Felino Barroso, n. 643, Fátima, Fortaleza, CE. CEP: 60050-130. 